\title{
Editorial
}

\section{Nanomaterials for Medical and Dental Applications}

\author{
Tae-Yub Kwon, ${ }^{1}$ Daniel S. Oh, ${ }^{2}$ and Ramaswamy Narayanan ${ }^{3}$ \\ ${ }^{1}$ Department of Dental Biomaterials, School of Dentistry, Kyungpook National University, Daegu 41940, Republic of Korea \\ ${ }^{2}$ College of Dental Medicine, Columbia University, New York, NY 10032, USA \\ ${ }^{3}$ School of Mechanical and Building Sciences (SMBS), VIT University, Tamil Nadu 600 127, India
}

Correspondence should be addressed to Tae-Yub Kwon; tykwon@knu.ac.kr

Received 12 December 2015; Accepted 13 December 2015

Copyright (C) 2015 Tae-Yub Kwon et al. This is an open access article distributed under the Creative Commons Attribution License, which permits unrestricted use, distribution, and reproduction in any medium, provided the original work is properly cited.

Welcome to this special issue. Nanoscience and nanotechnology concepts are applicable across all fields of science and a more widespread application of nanomaterials and nanotechnologies is imminent or already occurring in many areas, including health care. Today's scientists take those cutting-edge technologies and concepts and apply them to medicine and dentistry. They are finding a wide variety of ways to make medical and dental materials at the nanoscale to take advantage of their enhanced physical and biological properties.

The purpose of this special issue is to publish high-quality research papers as well as review articles addressing recent advances in the field of nanomaterials for medical and dental applications. A particular interest is given to papers exploring or discussing nanomaterials and nanotechnologies related to delivery system, bonding substitutes, and surface modification techniques applicable in these areas. For this special issue, several investigators were invited to contribute original research findings that can stimulate continuing efforts to understand the cutting-edge applications of nanomaterials in medicine and dentistry.

The physiological interaction between the titanium (Ti) surface and bone cells, especially osteoclasts, is a crucial factor in determining successful osseointegration. However, the details of such an interaction remain elusive. S. Oh et al. demonstrated that nanotopography on the Ti surface is a crucial factor for modulating intracellular signal transduction in bone marrow-derived macrophages (BMMs). In their study, UV photocatalysis of $\mathrm{TiO}_{2}$ immediately elicited $\left[\mathrm{Ca}^{2+}\right]_{\mathrm{i}}$ increase and $[\mathrm{ROS}]_{\mathrm{i}}$ reduction in cells growing on $\mathrm{TiO}_{2}$ nanotubes. UV photocatalysis-mediated $\left[\mathrm{Ca}^{2+}\right]_{\mathrm{i}}$ increase was dependent on extracellular and intracellular ROS generation. Furthermore, extracellular $\mathrm{Ca}^{2+}$ influx through VGCCs was critical for UV photocatalysis-mediated $\left[\mathrm{Ca}^{2+}\right]_{i}$ increase, while PLC activation was not. They concluded that nanotopography on the Ti surface should be considered an important factor that can influence successful dental implantation.

Mucinl (MUC1) is a potentially important target of cancer therapy, being a glycoprotein that is overexpressed on cell surface of many types of adenocarcinomas. Several MUC1targeted drug delivery systems have been developed and reported, but mobilizing natural killer cells (NK) to fight against MUC1-positive tumor has not been attempted. L. $\mathrm{Yu}$ et al. introduced a novel amphipathic nanoparticle for enhancing the NK cytotoxicity to MUC1-positive cancer cells. In their study, a novel amphipathic nanoparticle was constructed by implanting CD16 and MUC1 aptamers onto its surface. This nanoparticle pulled NK (or other CD16-positive immune cells) and MUC1-positive cancer cells together in vitro and significantly enhanced the NK cytotoxicity to MUC1-positive cancer cells. The authors suggested that NK cells might be mobilized to target against MUC1-positive tumor cells.

It has been suggested that the presence of white-spot lesion is very probable when adjacent surface is affected by cavitated lesions. C. A. B. Guglielmi et al. evaluated the potential of different fluoride-releasing restorative materials in arresting enamel white-spot lesions in approximal contact with them, in vitro (I) and in situ (II). White-spot lesions were formed in 120 primary enamel specimens via $\mathrm{pH}$-cycling and put in contact with cylindrical blocks of six different dental restorative materials. At the end of both studies, specimens 
were collected for mineral analysis by cross-sectional microhardness. High-viscous glass ionomer cements (HVGICs) were the most efficient in preventing mineral loss, whereas other materials presented an intermediate behavior. The authors concluded that fluoride-releasing materials can moderately reduce white-spot lesions progression, and HVGIC can arrest enamel lesion when in approximal contact.

Conventional scaffold fabrication methods do not control the architecture, pore shape, porosity, or interconnectivity of the scaffold, so it has limited ability to stimulate cell growth and to generate new tissue. In the review article, J. W. Lee suggests that three-dimensional (3D) printing technologies may overcome these disadvantages of traditional fabrication methods. These technologies use computers to assist in design and fabrication, so the 3D scaffolds can be fabricated as designed and standardized. Recent combinations of $3 \mathrm{D}$ nanoprinting technologies with methods from molecular biology and cell dynamics have suggested new possibilities for improved tissue regeneration. The author speculated that 3D nanoprinting will become an important tool in tissue engineering in the near future.

S. Jia et al. evaluated a novel, simple, and convenient method to enhance hydrophilicity of $\mathrm{Ti}$ surfaces with sodium bicarbonate $\left(\mathrm{NaHCO}_{3}\right)$ solution. Sand-blasted and acid-etched (SLA) Ti surfaces and machined Ti surfaces were treated with $\mathrm{NaHCO}_{3}$ solution. In their study, superhydrophilic SLA Ti surfaces were obtained by reversible deprotonation and ion exchange processes. Increased protein adsorption on $\mathrm{NaHCO}_{3}$-treated specimens was also observed. They concluded that this $\mathrm{NaHCO}_{3}$ treatment is a reliable method for enhancing the hydrophilicity and protein adsorption of SLA Ti surfaces.

V. Babuska et al. compared the in vitro response of human fibroblast cell line HFLl and human osteoblast cell line hFOB 1.19 on nanostructured $\mathrm{Ti}$ with different grain size. In their study, similar cellular behavior was observed on all studied biomaterials. There were significant differences related to the initial phase of attachment, but not in proliferation. Furthermore, the results indicate that osteoblasts grow the best on material with grain size of $160 \mathrm{~nm}$ with a longitudinal section in comparison with other examined materials. The authors concluded that this material could be recommended for further evaluation with respect to osseointegration in vivo.

Basic calcium phosphate (BCP) crystals have been associated with many diseases due to their activation of signaling pathways that lead to their mineralization and deposition in intra-articular and periarticular locations in the bones. In the study by $\mathrm{C}$. Herdocia-Lluberes et al., hydroxyapatite (HAp) was placed in a polysaccharide network as a strategy to minimize the BCP cell activation. Their research consisted of the evaluation of varying proportions of the polysaccharide network, cellulose nanocrystals (CNCs), and HAp synthesized via a simple sol gel method. After preparation of the biocompatible composite, the products were characterized by various analytical methods. It was found that a nHAp = $\mathrm{CNC}$ ratio presented greater homogeneity in the size and distribution of the nanoparticles without compromising the crystalline structure. The osteoblast cell viability assay also demonstrated that all of the composites promoted greater cell proliferation. The authors suggested that the nHAp $>\mathrm{CNC}$ proportion with the inclusion of the bone morphogenetic protein 2 (BMP-2) protein was the best composite.

Tae-Yub Kwon

Daniel S. Oh

Ramaswamy Narayanan 

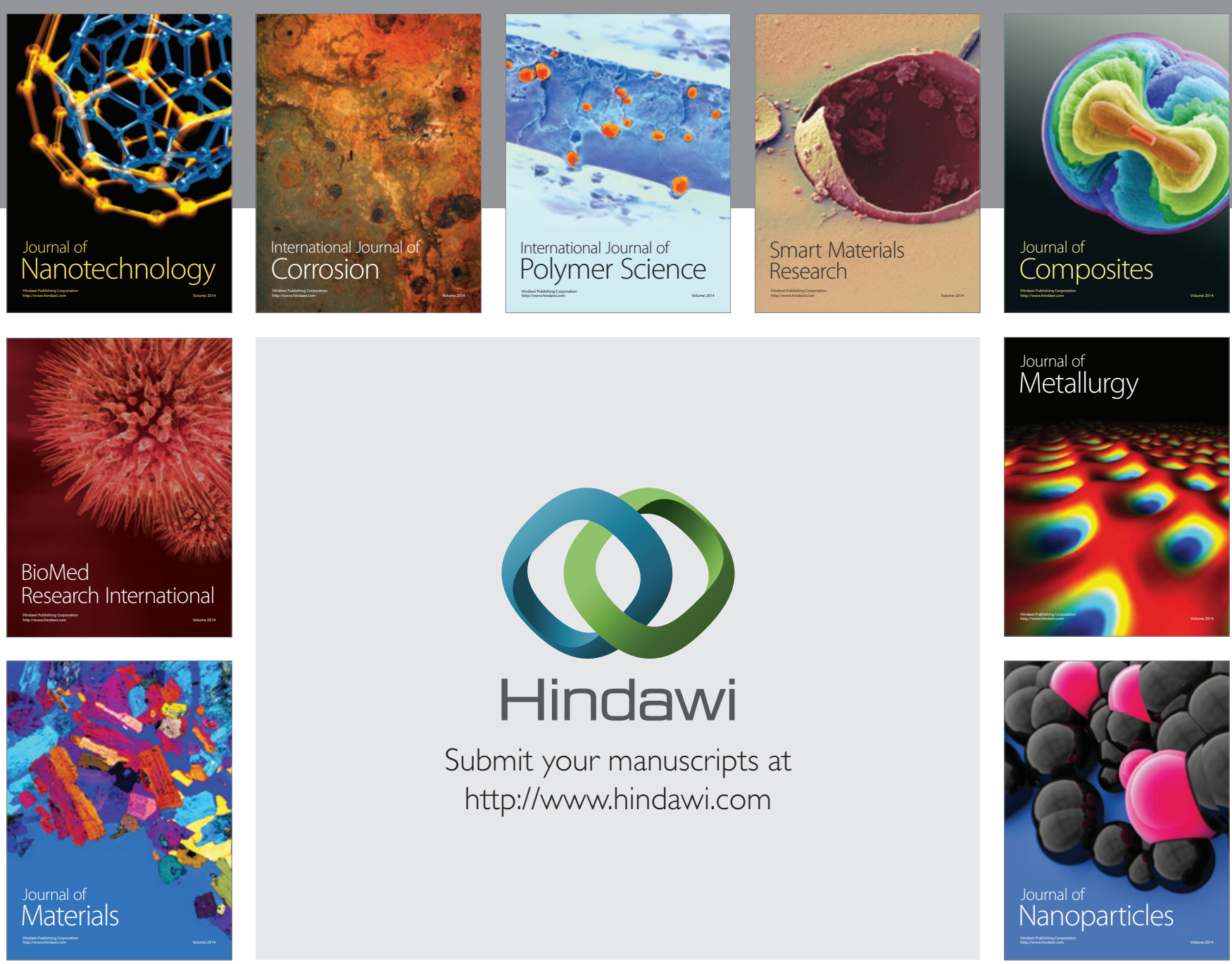

Submit your manuscripts at http://www.hindawi.com
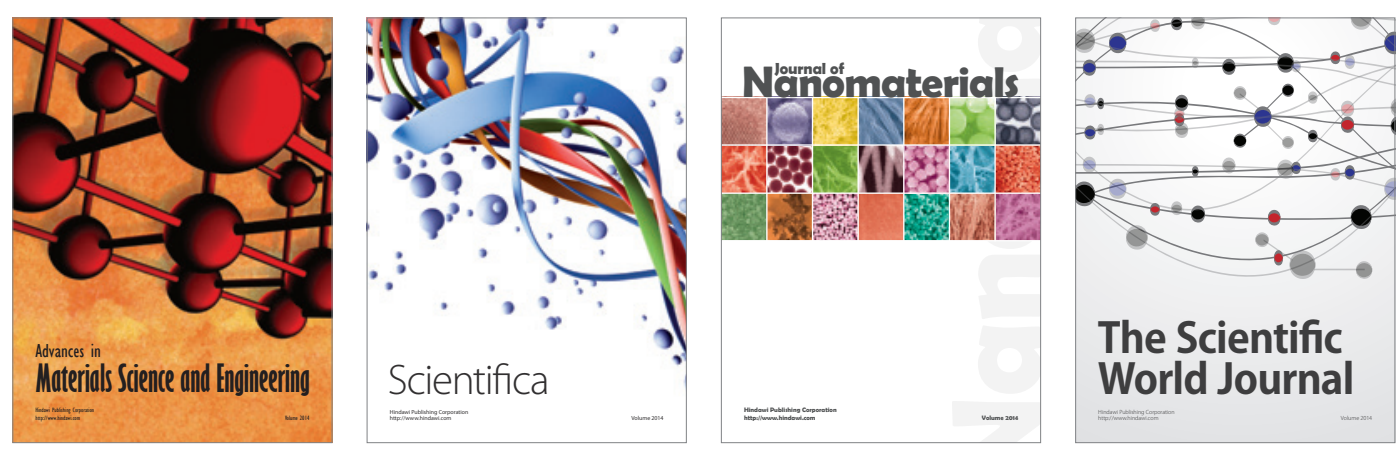

\section{The Scientific World Journal}
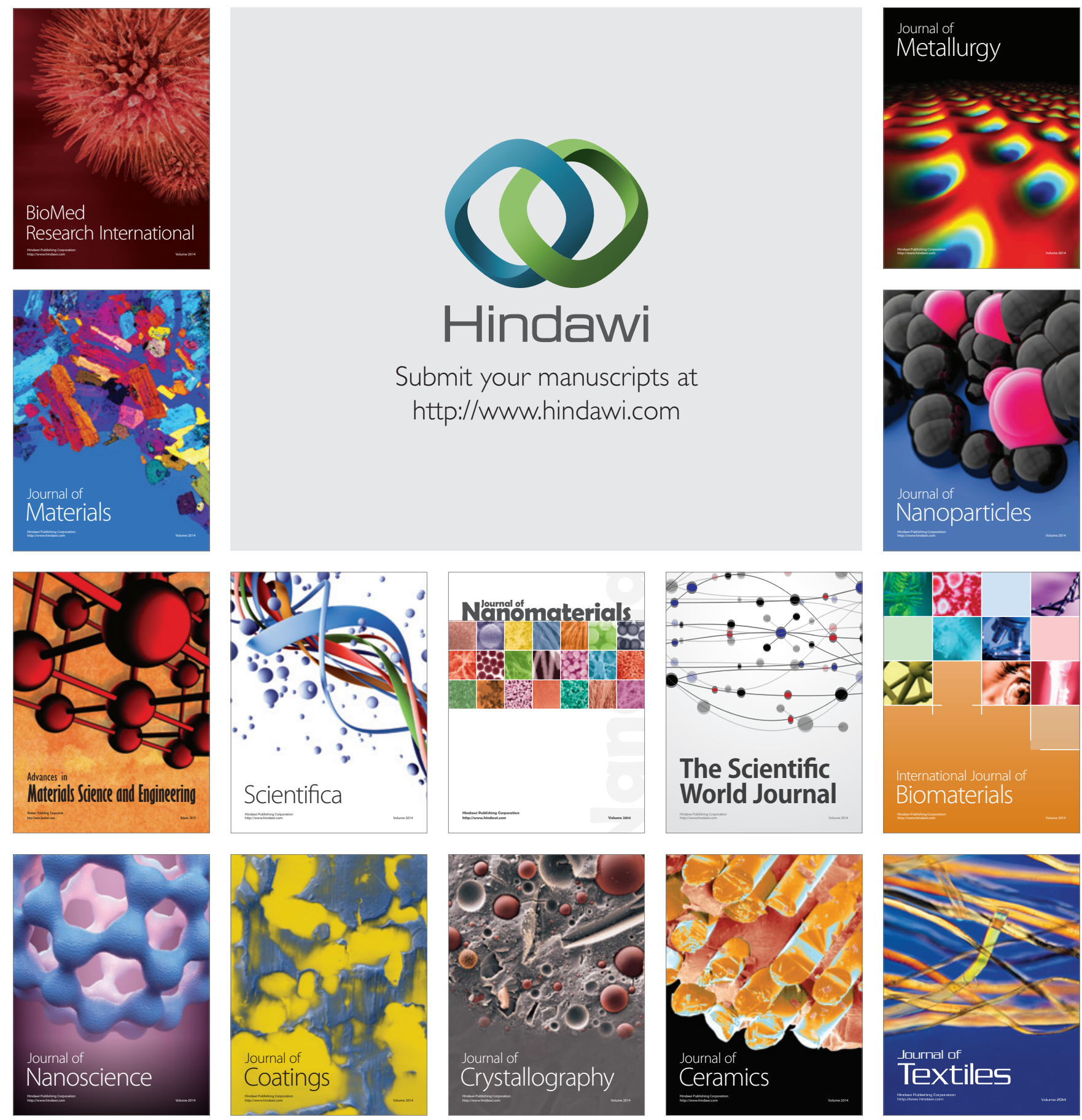